\title{
Remarks on Parameter Estimation for Computational Model of Plasma-Organic-Polymer-Film-Coated QCR Sensor
}

\author{
Kazuhiko Takahashi $^{\dagger, *}$, Ryosuke $\mathrm{Kita}^{\dagger}$, Iwao Sugimoto ${ }^{\ddagger}$ \\ $\dagger$ Doshisha University, 1-3 Miyakodani Tatara Kyotanabe Kyoto, Japan \\ ${ }^{\ddagger}$ Tokyo University of Technology, 1404 Katakura, Hachioji, Tokyo, Japan \\ *Corresponding Author: katakaha@mail.doshisha.ac.jp
}

\begin{abstract}
This paper investigates a computational model of the masssensitive chemical sensor using plasma-organic-polymerfilm-coated quartz crystal resonators and discusses the model's parameter estimation based on a steepest descent method. Diffusion equations are applied sample gas molecules in the neighbourhood space of the sensor and the bulk of a sensor film to describe the responses of the chemical sensor to the sample gas. Laplace transform is utilized to solve the governing equations, and the computational model of the chemical sensor response is derived. Computational experiments of the chemical sensor response are conducted to compare the sensor responses calculated by the proposed model with the experimental results obtained by the gas sensing system using a natural herb and spice as sample gas sources. The experimental results confirm the feasibility of the proposed method to estimate the responses of the chemical sensor.
\end{abstract}

Keywords: Plasma organic polymer film, Quartz crystal resonator, Diffusion equation, Laplace transform.

\section{Introduction}

An electronic nose based on chemical sensor technologies is expected in various practical applications, such as monitoring indoor air quality, investigating the freshness/staleness of foods and diagnosing human health/activity with biogas. $^{(1)-(4)}$ To develop a high-performance electronic nose system, many studies on chemical materials, sensor devices, sensor systems and their signal processing algorithms have been conducted. The authors have also presented gas monitoring applications ${ }^{(5)}$ that use mass-sensitive chemical sensors based on an array of quartz crystal resonators (QCRs), which are coated with plasma organic polymer films (POPFs $^{(6)}$ and investigated a computational model of the POPF-coated QCR sensor ${ }^{(7)-(10)}$ to estimate the response of sensor dynamics for designing signal processing algorithm in intelligent electronic nose systems.

In this paper, we discuss a parameter estimation method for the computational model of the POPF-coated QCR sensor. The well-known Fickian diffusion equation is used to describe the computational model of representing the concentration of sample gas molecules in the neighbourhood space of the sensor and the bulk of a sensor film. In computational experiments, the parameters of the computational model is estimated using a steepest descent method so as to fit the computational model with the sensor responses obtained with a gas-sensing system using the POPF-coated QCR sensors.

\section{Computational Model of POPF-coated QCR Sensor}

Figure 1 illustrates the diffusion model of the sample gas molecules in the neighbourhood space of the sensor (Area I) and the bulk (Area II) of a sensor film. The dynamics of molecule concentration, $\psi_{i}\left(x_{i}, t\right)(i=1,2)$, at position $x_{i}$ and time $t$ can be given by

$$
\frac{\partial \psi_{i}\left(\boldsymbol{x}_{i}, t\right)}{\partial t}=D_{i} \nabla^{2} \psi_{i}\left(\boldsymbol{x}_{i}, t\right)-\operatorname{div}\left(\boldsymbol{v}_{i} \psi_{i}\left(\boldsymbol{x}_{i}, t\right)\right)
$$

where $D_{i}(i=1,2)$ is the effective diffusion coefficient in each area, $\boldsymbol{v}_{1}$ is the velocity of the gas flow and $\boldsymbol{v}_{2}$ is the velocity of the QCR vibration. Here, the QCR vibrates at an in-plane sliding mode. To simplify the analysis, we assume that the concentration gradients with respect to the position in $y_{i}$ and $z_{i}$ axes, as well as those with respect to the velocity, are zero. Thus, the one-dimensional diffusion equations can be obtained in each area. The boundary conditions are

$$
\begin{aligned}
\psi_{1}(0, t) & =f(t), \\
\frac{\partial \psi_{1}\left(L_{1}, t\right)}{\partial x_{1}} & =\frac{\partial \psi_{2}(0, t)}{\partial x_{2}}, \\
\psi_{2}(0, t) & =h(t), \\
\frac{\partial \psi_{2}\left(L_{2}, t\right)}{\partial x_{2}} & =0,
\end{aligned}
$$

where $f(t)$ expresses the input pattern of the arbitrary sample gas concentration, $L_{1}$ is the distance between the gas

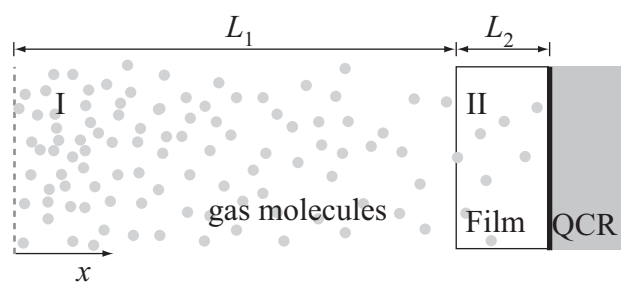

Fig. 1. Diffusion process model of the chemical sensor. 
source and upper film boundary, $L_{2}$ is the sensor film thickness and $h(t)$ represents the dynamics of sample gas sorption/desorption at the sensor film surface as follows:

$$
a \frac{d h(t)}{d t}+b h(t)=c \psi_{1}\left(L_{1}, t\right),
$$

where $a, b$ and $c$ are the model parameters. The initial conditions are $\psi_{i}\left(x_{i}, 0\right)=\psi_{i_{0}}$. By applying the Laplace transform to the governing equations, the boundary conditions and the first-order model, we can obtain the solutions of the diffusion equations. Due to the relationship between the resonant frequency shift (RFS) of the QCR and the the mass variation of the sensor film, the sensor response is obtained by calculating the mass of the sample gas molecule in the sensor film bulk as follows:

$$
m(t)=\int_{0}^{L_{2}} \psi_{2}\left(x_{2}, t\right) d x_{2} .
$$

\section{Computational Experiment of Chemical Sensor Response}

The diffusion coefficient is the important parameter to define the response of the chemical sensor, however, that in the material of POPFs has not yet been reported. In this study, the diffusion coefficient of the POPF is approximated using a capillary model as follows:

$D_{2}=p_{1} D_{s e}\left(1-p_{2}\right)^{2} \frac{1-2.1 p_{2}+2.1 p_{2}^{3}-1.7 p_{2}^{5}+p_{2}^{6}}{1-0.76 p_{2}^{5}}$,

where $p_{1}$ is the ratio between the porosity of the POPF and the tortuous factor, and $D_{s e}$ is the diffusion coefficient of the spherical particles in the molecule given by the StokesEinstein equation $D_{s e}=k_{B} T /(6 \pi \mu r)$ where $k_{B}$ is the Boltzmann's constant, $T$ is the absolute temperature, $\mu$ is the viscosity, $r$ is the spherical particle radius and $p_{2}$ is the ratio between the pore radius $R$ and the spherical particle radius. The pore radius, porosity and tortuous factor are unknown. Therefore, the model parameters $D_{s e}, p_{1}$ and $p_{2}$ are tuned using a steepest descent method so as to fit the computational model with the sensor responses obtained with the gas sensing system shown in Fig. 2 where a natural herb/spice was used as sample gas source and seven sensor films were coated with D-phenylalanine (s1), D-tyrosine (s2), D-glucose (s3), DL-histidine (s4), adenine (s5), polyethylene (s6) and polychlorotrifluoroethylene (s7). In the steepest descent method, the cost function is defined as follows:

$$
J=\frac{1}{2} \sum_{t=0}^{t_{e}}\{\Delta \bar{F}(t)-\bar{m}(t)\}^{2},
$$

where $\Delta \bar{F}(t)$ is the RFS of the experimental sensor response normalized with the maximum RFS and $\bar{m}(t)$ is the response of the computational model normalized with the final value $m(\infty)$. This is because that the RFS of the QCR is in proportion to mass variation of the sensor film. The parameters of the capillary model were updated by

$$
\boldsymbol{w}(p+1)=\boldsymbol{w}(p)-\gamma \frac{\partial J}{\partial \boldsymbol{w}(p)},
$$

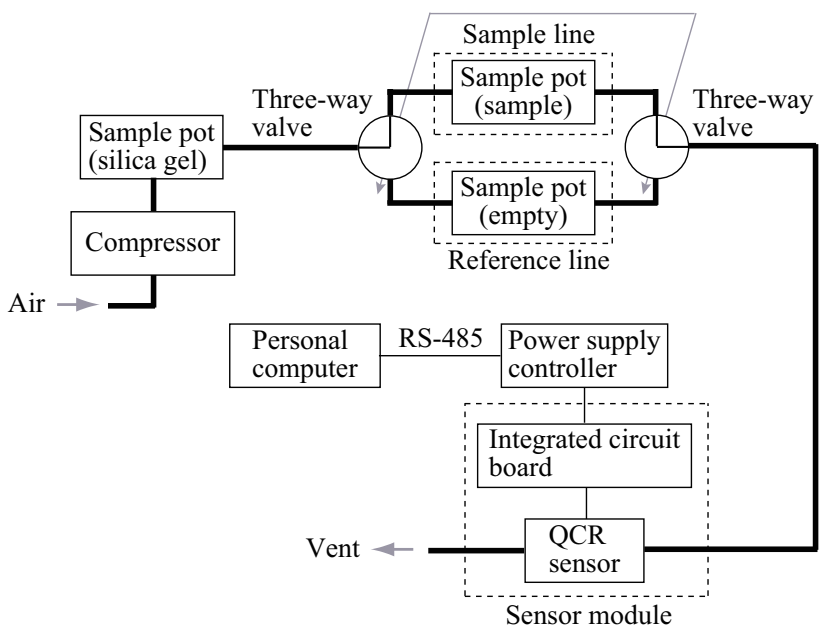

Fig. 2. Diagram of a gas-sensing system.

where $p$ is the iteration number, $\gamma$ is the step size and the vector $\boldsymbol{w}$ is composed of the parameters $D_{s e}, p_{1}$ and $p_{2}$. In the estimation process using the steepest descent method, the experimental data was sampled with the interval of 10 seconds to evaluate the cost function and the total number of the sample from each sensor was 90 . In the calculation of updating the parameter, the step sizes was $\gamma=0.05$ and the gradient of the cost function with respect to the diffusion coefficient was calculated using a finite difference approximation. The response of the computational model was calculated using the numerical inversion of the Laplace transform ${ }^{(11)}$ with the following conditions: the initial condition was set to 0 and $f(t)$ was a Heaviside function. The physical parameters of the gas-sensing system were $D_{1}=0.219 \times 10^{-4}$ (water vapour), $L_{1}=0.05, v_{1_{x}}=2.653 \times 10^{-3}, T=298$, $\mu=1 \times 10^{-3}, r=1.325 \times 10^{-10}$ (water molecule). In the sorption/desorption model, the coefficients were set to $a=1$, $b=1$ and $c=1$. The computational experiments were conducted using SCILAB version 5.5.0 (Scilab Enterprises).

Table 1 shows the estimation results of the diffusion coefficient. This result shows the possibility of classifying the sample gas using the estimated diffusion coefficients as a feature vector. Figures 3 and 4 compare the experimental result (black dot) with the computational model response (red line) after the convergence of the parameter estimation. Here the sample gas source is a natural herb of $2 \mathrm{~g}$ basil and the material of the sensor films are D-phenylalanine and polychlorotrifluoroethylene, respectively. Figures 5 and 6 show the results of computational experiment in which the sample gas source is a natural spice of $2 \mathrm{~g}$ cinnamon, Figs. 7 and 8 show the results of computational experiment in which the sample gas source is a natural herb of $2 \mathrm{~g}$ lemongrass and Figs. 9 and 10 show the results of computational experiment in which the sample gas source is a natural herb of $2 \mathrm{~g}$ oregano. As shown in these figures, the computational model can approximate the responses observed in the experimental results. These results show the feasibility of the proposed model of the POPF-coated QCR sensor. 
Table 1. Estimation results of the diffusion coefficient for natural herb/spice.

\begin{tabular}{|c|c|c|c|c|c|}
\hline sensor cell & $L(\AA)$ & \multicolumn{4}{|c|}{$D\left(\times 10^{-11} \mathrm{~m}^{2} / \mathrm{s}\right)$} \\
\cline { 3 - 6 } & & basil & cinnamon & lemongrass & oregano \\
\hline s1 & 9889 & 6.028204 & 6.686083 & 4.520859 & 5.445818 \\
\hline s2 & 8948 & 5.408789 & 6.269840 & 4.284238 & 4.677070 \\
\hline s3 & 11159 & 6.993911 & 9.083010 & 5.246158 & 6.425534 \\
\hline s4 & 9324 & 6.342177 & 9.008776 & 4.458653 & 5.507061 \\
\hline s5 & 8275 & 6.691403 & 12.02806 & 4.652143 & 5.211138 \\
\hline s6 & 11349 & 10.25561 & 15.02807 & 5.913031 & 8.051347 \\
\hline s7 & 5646 & 6.639306 & 13.63013 & 3.243723 & 4.945856 \\
\hline
\end{tabular}

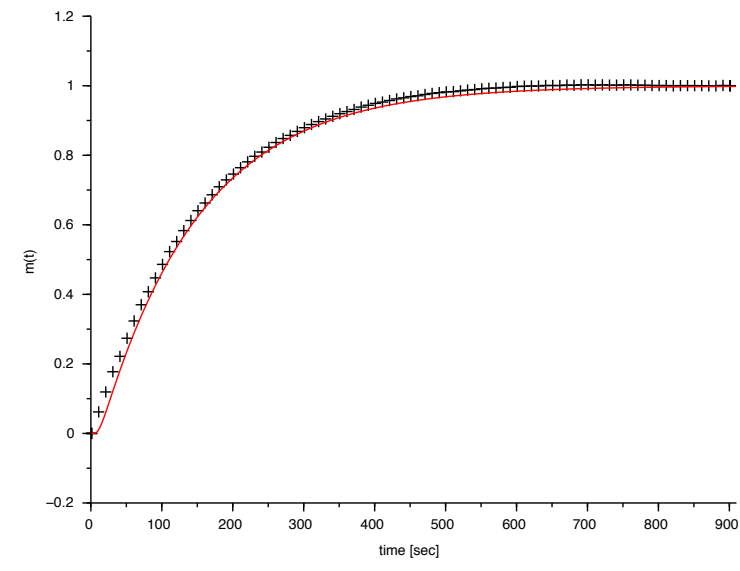

Fig. 3. Transient response of POPF-coated QCR sensor with D-phenylalanine (biomaterial) film for sample gas source of basil.

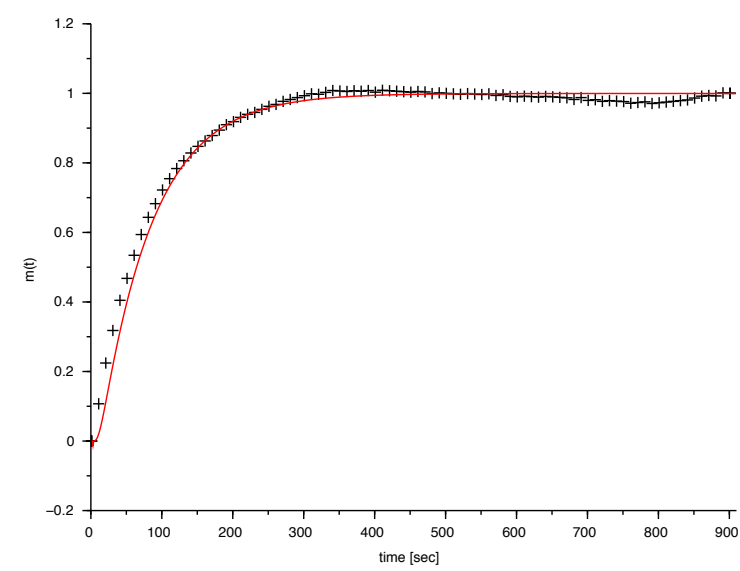

Fig. 4. Transient response of POPF-coated QCR sensor with polychlorotrifluoroethylene (synthetic polymer) film for sample gas source of basil.

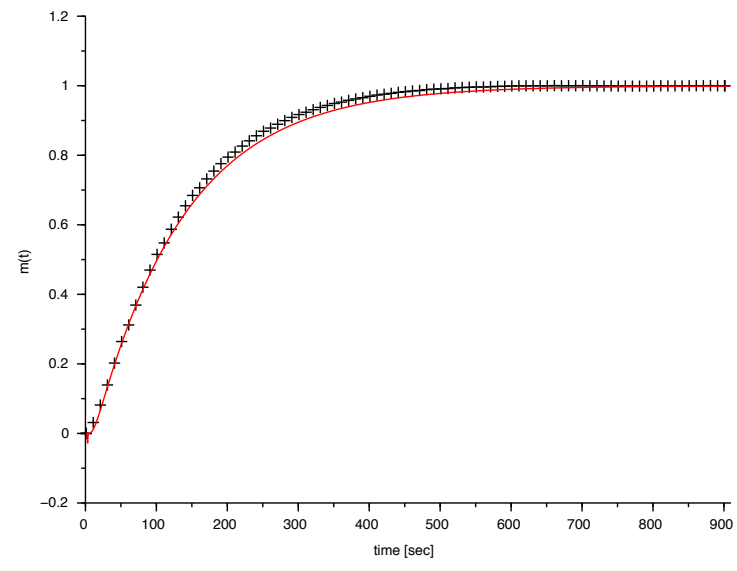

Fig. 5. Transient response of POPF-coated QCR sensor with D-phenylalanine (biomaterial) film for sample gas source of cinnamon.

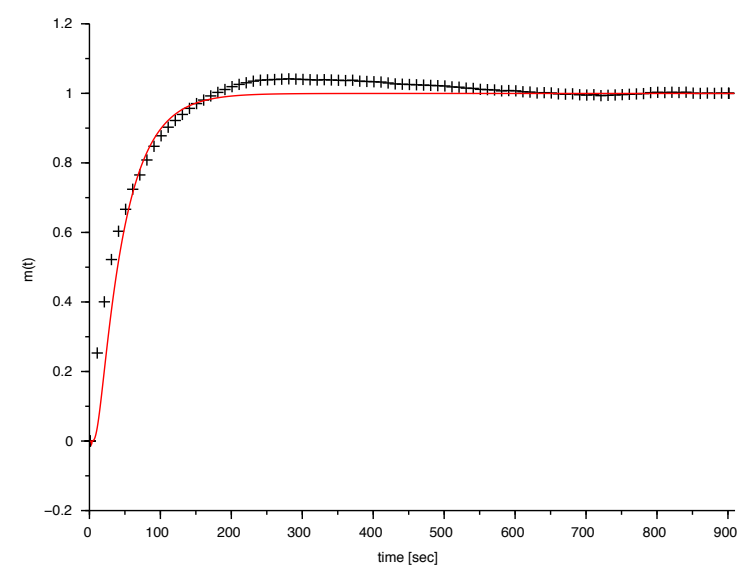

Fig. 6. Transient response of POPF-coated QCR sensor with polychlorotrifluoroethylene (synthetic polymer) film for sample gas source of cinnamon. 


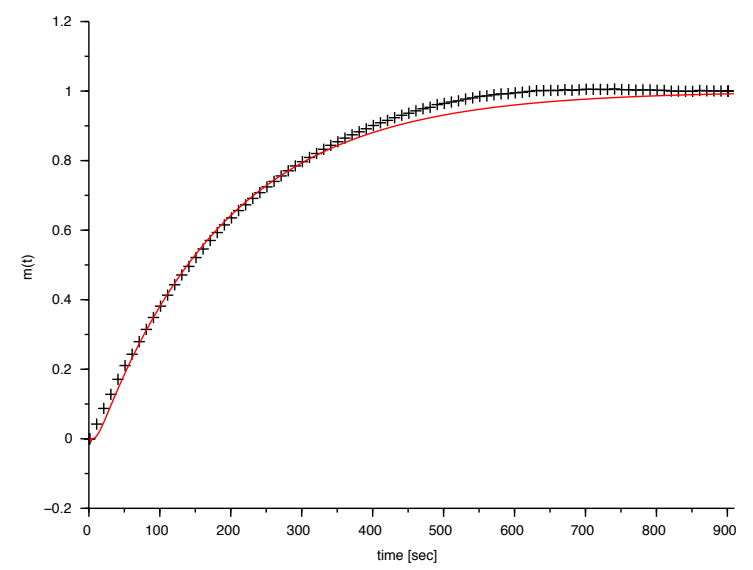

Fig. 7. Transient response of POPF-coated QCR sensor with D-phenylalanine (biomaterial) film for sample gas source of lemongrass.

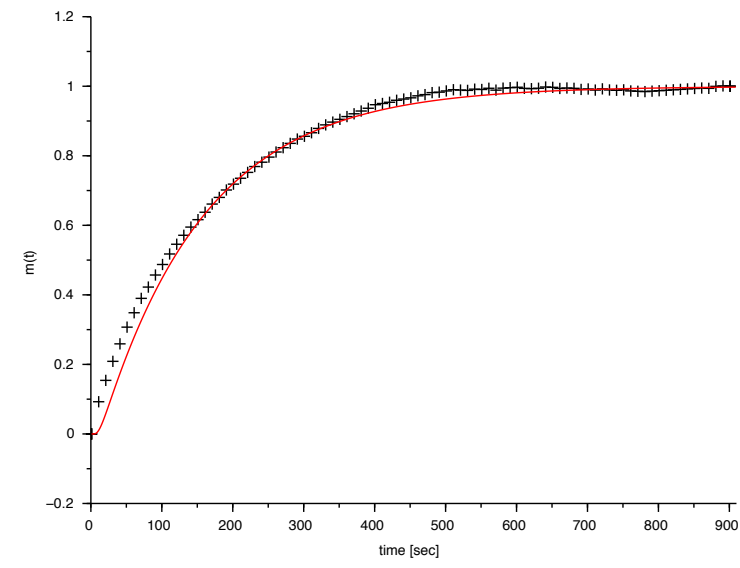

Fig. 8. Transient response of POPF-coated QCR sensor with polychlorotrifluoroethylene (synthetic polymer) film for sample gas source of lemongrass.

\section{Conclusions}

This paper investigated a computational model of the mass-sensitive chemical sensor with POPF-coated QCRs and introduced a steepest descent method to estimate the diffusion coefficient parameter in the computational model. To describe dynamic responses of the chemical sensor to sample gas molecules, Fickian diffusion equation was applied to the sample gas molecules and the computational model of the chemical sensor response was obtained by solving the governing equations using Laplace transform. The computational experiments, in which a natural herb and spice were used as sample gas sources, proved the feasibility of the proposed model in the estimation of the chemical sensor responses.

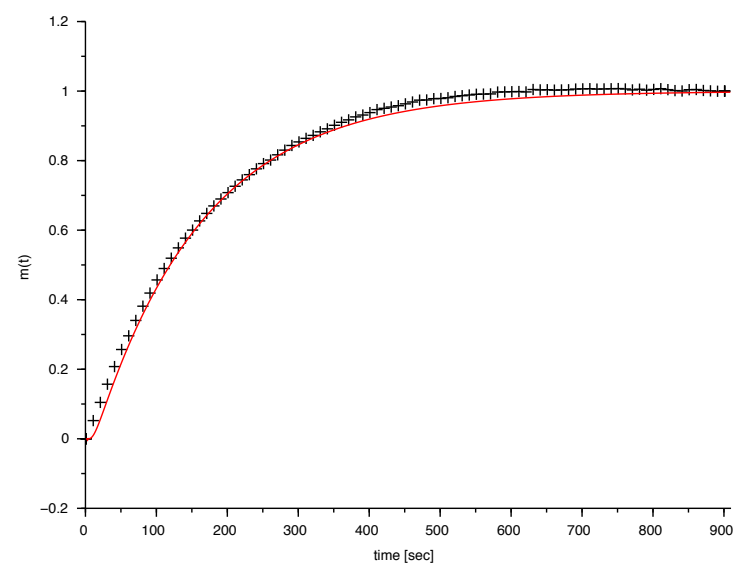

Fig. 9. Transient response of POPF-coated QCR sensor with D-phenylalanine (biomaterial) film for sample gas source of oregano.

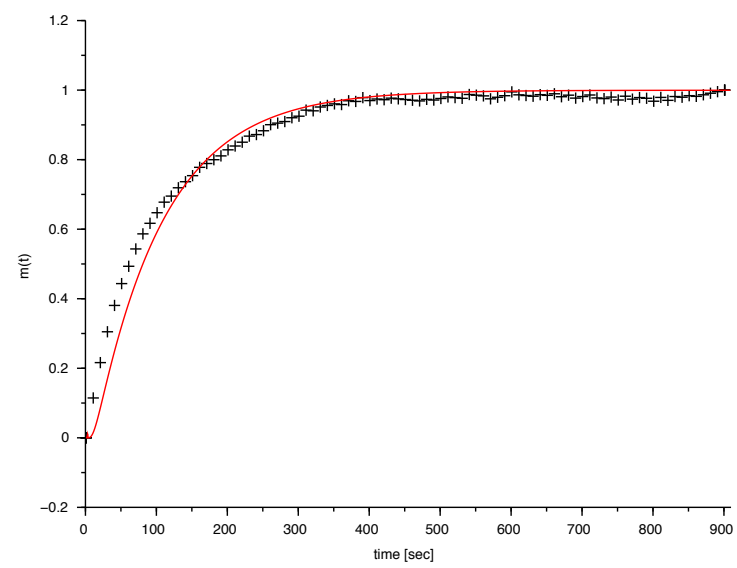

Fig. 10. Transient response of POPF-coated QCR sensor with polychlorotrifluoroethylene (synthetic polymer) film for sample gas source of oregano.

\section{Acknowledgment}

This research was partially funded by MEXT/JSPS KAKENHI Grant no. 24560863.

\section{References}

(1) A. D. Wilson and M. Baietto: "Applications and Advances in Electronic-Nose Technologies", Sensors, Vol. 9, pp. 5099-5148, 2009.

(2) E. A. Baldwin, J. Bai, A. Plotto and S. Dea: "Electronic Noses and Tongues: Applications for the Food and Pharmaceutical Industries", Sensors, Vol. 11, pp. 4744-4766, 2011. 
(3) H. Alam and S. H. Saeed: "Electronic Nose in Food and Health Applications: A Review", International Journal of Computing and Corporate Research, Vol. 2, No. 6, 2012.

(4) A. D. Wilson: "Review of Electronic-nose Technologies and Algorithms to Detect Hazardous Chemicals in the Environment", Procedia Technology, Vol. 1, pp. 453-463, 2012.

(5) K. Takahashi and I. Sugimoto: "Remarks on Emotion Recognition Using Breath Gas Sensing System”, Smart Sensors and Sensing Technology, Lecture Notes Electrical Engineering, Vol. 20, pp. 49-62, 2008.

(6) I. Sugimoto, M. Nagaoka, M. Seyama, M. Nakamura and K. Takahashi: "Classification and Characterization of Atmospheric VOCs based on Sorption/Desorption Behaviors of Plasma Polymer Films", Sensors and Actuators (B: Chemical), Vol. 124, pp. 53-61, 2007.

(7) K. Takahashi, Y. Iwamuro and I. Sugimoto: "Analysis of Dynamic Response of Plasma Polymer Film-Coated Quartz Crystal Resonator Sensor", International Journal of Applied Electromagnetics and Mechanics, Vol. 41, No. 1, pp. 39-49, 2013.

(8) K. Takahashi, Y. Kawanobe and I. Sugimoto: "Computational Analysis of the Dynamics of Plasma-OrganicPolymer-Film-Coated QCR Sensors Using Numerical Inversion of a Laplace Transform", in Proceedings of the Second International Conference on Computing, Measurement, Control and Sensor Network, CD-ROM20, 2014.

(9) K. Takahashi, N. Nishiwaki and I. Sugimoto: “Computational Model of Plasma-Organic-Polymer-FilmCoated QCR Sensor For Analysing Its Dynamic Response", in Proceedings of the 8th International Conference on Sensing Technology, pp. 85-88, 2014.

(10) K. Takahashi, Y. Kawanobe, N. Nishiwaki and I. Sugimoto: "Remarks on a Computational Model of a Mass-Sensitive Chemical Sensor with PlasmaOrganic-Polymer-Film-Coated Quartz Crystal Resonators", in Proceedings of 2014 IEEE/SICE International Symposium on System Integration, pp. 558-563, 2014.

(11) T. Hosono: "Numerical Inversion of Laplace Transform and Some Application to Wave Optics", Radio Science, Vol. 16, No. 6, pp. 1015-1019, 1981. 